\title{
LAS REPRESENTACIONES SOCIALES DE PROFESORES Y PROFESORAS SOBRE LA INCLUSIÓN DE NIÑOS Y NIÑAS INDÍGENAS Y CON DISCAPACIDAD EN DOS ESCUELAS DE EDUCACIÓN PRIMARIA DE OAXACA, MÉXICO
}

\author{
THE MEANINGS ATTRIBUTED BY TEACHERS TO THEIR PEDAGOGICAL \\ PRACTICE WITH CHILDREN WITH PHYSICAL AND INTELLECTUAL \\ DISABILITIES IN TWO SCHOOLS OF INDIGENOUS PRIMARY EDUCATION \\ IN OAXACA, MEXICO
}

Gloria Rosario Victoria Avila

Especialista en educación indígena y discapacidad

gloriavictoria26@gmail.com

\begin{abstract}
Resumen: Las niñas y niños indígenas con discapacidad hoy día son sujetos de derecho. Diferentes documentos nacionales e internacionales reconocen los derechos de las personas con discapacidad para su acceso y participación en los diferentes ámbitos sociales, como el acceso a la educación inclusiva en los sistemas de las escuelas indígenas regulares. El estudio se centró en el paradigma cualitativo desde un enfoque comprensivo interpretativo, lo que permitió captar y develar la realidad social de los docentes mediante entrevista en profundidad. Los hallazgos encontrados están centrados en las representaciones de los docentes, a partir de las experiencias para atender a los niños y niñas en situación de discapacidad, algunas de ellas: escasa formación profesional y capacitación recibida, las dificultades en su práctica pedagógica, las posibles causas atribuidas del padecimiento que sufren los educandos, las características de las dificultades que ellos y ellas portan, el contexto familiar en las comunidades indígenas. Asimismo, las dificultades que tienen ante el desafío de tener alumnos/as en el espacio áulico regular.
\end{abstract}

Palabras clave: representaciones de los docentes, discapacidad, educación inclusiva.

Abstract: Today, indigenous children with disabilities are subjects of rights. Several national and international documents recognize the rights of people with disabilities to access and participate in different social places, such as accessing to inclusive education in regular indigenous schools. The study focuses on the qualitative paradigm from a comprehensive interpretive approach, which allowed capturing and unveiling a teachers' social reality through in-depth interviews. The findings found are focused on teachers' representations, based on experiences to attend to children with disabilities. Some of those are: lack of professional training and training received difficulties in their pedagogical practice, possible causes attributed to the suffering suffered by the students, the characteristics of the difficulties they carry, family context in the indigenous communities. Also, it shows the difficulties they face in the challenge of having students in the regular classroom.

Key words: teachers' representations, disability, inclusive education. 


\section{INTRODUCCIÓN}

La diversidad socio-cultural que existe en México ha implicado un gran desafío para el país, pues la mayoría de las poblaciones se encuentran en territorios de difícil acceso, lo que ha impedido que el Estado conozca las diferentes problemáticas que existen en las comunidades indígenas, la ofertan de programas para la educación inclusiva en la discapacidad.

Por lo tanto, una de las situación que aun parece encontrarse en invisibilidad para el gobierno es la realidad en la que se encuentran las personas indígenas con discapacidad, si bien la situación ha sido compleja comparada a otros grupos, más aún, cuando se trata de las niñas y niños indígenas con discapacidad pues sufren doble vulneración para su atención, participación e inclusión en la comunidad de pertenencia y en la escuela se necesita el trabajo colaborativo de todas las instancias para poner en marcha políticas y programas, que permitan llevar educación de calidad en todos los rincones del país y llegar a aquellos niños y jóvenes que más lo necesitan y que tradicionalmente han sido excluidos del sistema.

\section{DESARROLLO}

En México de los 68 grupos indígenas que existen la mayoría se encuentra en una desigualdad educativa. Al respecto una de las situaciones que actualmente está en continuo debate entre los pueblos originarios es el tema de la educación que el Estado les imparte, por generaciones la educación indígena siempre se ha referido a aquello que se considera que los pueblos deben saber, una apreciación externa a su cultura, homologando a todos los ciudadanos. Pero ¿Por qué es importante una educación indígena de calidad y equidad para los pueblos originarios? Según Delors (1994), dice en su obra. La educación encierra un tesoro. La educación es un proceso que se da a lo largo de toda la vida. Cumple dos funciones principales: transmite los conocimientos acumulados en relación con el mundo y a su vez, habilita a los miembros de la sociedad para vivir en un mundo en constante cambio. Se trata, además, de un proceso de socialización, dado que el habitar en ese mundo cambiante se da siempre en interacción con otros miembros de la sociedad. Asimismo esto da la posibilidad de que a través de ella se pueden obtener los conocimientos y aptitudes necesarios para que las actuales generaciones puedan desenvolverse individual, familiar y socialmente en cualquier contexto.

Si bien, desde la Constitución Política de los Estados Unidos Mexicanos se reconoce que cualquier sujeto tiene el derecho de acceder a ella sin importar su condición de género, raza, religión o con algún tipo de discapacidad etc. así en su artículo 3 reconoce que "Todo individuo tiene derecho a recibir educación, la educación que imparta el Estado tenderá a desarrollar armónicamente, todas las facultades del ser humano" (DOF, 2016, p.4). Desde esta mirada vemos lo importante que es acceder a la educación desde un enfoque en derechos humanos, inclusión y calidad que permita la plena participación, la 
identidad y el reconocimiento de las capacidades propias del sujeto para su desarrollo integral.

Aunque, los niños y niñas indígenas encuentran dificultades para acceder a una educación apropiada a su contexto dentro de las comunidades, dentro de este grupo se encuentra otros grupos mucho más desfavorecido como es el caso de los niños (as) con discapacidad, por el medio social en la que viven se enfrentan a obstáculos adicionales, por ser indígenas y con discapacidad, históricamente, han sido uno de los grupos con mayor dificultades para acceder a la educación indígena, actualmente persisten numerosas barreras físicas y culturales que obstaculizan su ingreso y tránsito a través del sistema educativo.

Pero ¿quiénes son las personas con discapacidad? En diferentes culturas y épocas eran conocidos como: minusválidos, deficientes, anormales, etcétera, son algunas de las fórmulas que se han empleado para adjetivar a las personas con discapacidad, el modo en que se nombra o se hace referencia a los seres $u$ objetos se encuentra fuertemente vinculado con el orden de significaciones que imperan en una época y en una sociedad determinada (Soto, 2011).

Por esta razón es importante conocer en la actualidad cuales son las representaciones sociales que tienen acerca de las personas con discapacidades en México. Al respecto la discapacidad "es entendida como una deficiencia física, intelectual o mental ya sea permanente o temporal, que al interactuar con el contexto tiene barreras para una participación plena" (DOF, 2015.). Es relevante de algún modo, dado que las posibles significaciones apertura a las condiciones actuales de exclusión/inclusión y aceptación/rechazo de las personas con discapacidad.

Esta representación que ha tenido la sociedad en torno a las personas con discapacidad también ha influenciado en las diversas formas de considerar su participación en la sociedad como: educativas, laborales y sociales, aunque el Estado a lo largo de los años ha tratado de crear diferentes políticas, instituciones y programas reconociendo los derechos de las personas con discapacidad, por su parte para los pueblos originarios la situación de las personas con discapacidad es mucho más compleja por el medio geográfico en la que se encuentran, por las condiciones sociales, económicos y educativos en la que viven, los servicios no llegan, los programas no son los adecuados para responder sus necesidades, así como la forma de entender y tratar los males corporales y su posible sanación por medio de prácticas interculturales que tiene la comunidad.

Por lo que respecta "la escuela, y el sistema educativo en su conjunto, son una instancia de mediación cultural entre los significados, sentimientos y conductas de la comunidad social y el desarrollo particular de nuevas generaciones" (Pérez, 2004, p.11) y tiene como misión fundamental contribuir a la mejora de la sociedad a través de la formación de ciudadanos críticos, responsables y honrados (Guerra, 2006). Asimismo, 
como una institución de formación debe aceptar a alumnos con diferentes condiciones sociales, diversidad cultural, como las niños/as y jóvenes con discapacidad, la institución debe ser flexible ante los diversos problemas que viven y enfrentan los alumnos; sin embargo esto no es lo que sucede en las instituciones.

Ante esto, los factores de exclusión asociados con la oferta educativa son: prácticas discriminatorias, estigmas y maltratos en la escuela, porque aún en los pueblos originarios prevalecen escuelas que no aceptan a alumnos por su condición de discapacidad. Según Instituto Nacional de Estadística y Geográfica [INEGI] (2010), en México las personas que tienen algún tipo de discapacidad representan $5.1 \%$ de la población total, y el $6.6 \%$ corresponde a personas indígenas, en el caso del Estado de Oaxaca ocupa el $8^{\circ}$ lugar, con mayor población en discapacidad por lo que puede presentar mayor vulnerabilidad, debido a su doble condición: con discapacidad e indígena.

En el informe perfil sociodemográfico de la población con discapacidad en el Estado de Oaxaca (2010), menciona que es un Estado con gran diversidad étnica, de tal manera que de las 8 regiones que compone, la región de la Mixteca es la que tiene el mayor porcentaje de población con discapacidad teniendo un $7.63 \%$. Además, la probabilidad de que viva en el sector rural le genera otra desventaja, son lugares que tienen escasos y deficientes servicios educativos. Los profesores enfrentan grandes retos para atender los desafíos del presente y el desarrollo del futuro, pues la mayoría vive en comunidades alejadas de los centros urbanos $y$, generalmente, pertenecen a un pueblo originario. Tienen como reto trabajar para atender las demandas y necesidades educativas de las niñas y niños indígenas con y sin discapacidad.

En este estudio se pretende abordar sobre las niñas y niños con dificultad cognitiva e intelectual que no han logrado una plena inclusión en dos escuelas regulares de la comunidad de Santa Cruz Nundaco ubicado en Oaxaca. Entendiendo que la inclusión según (Macuralla y Saiz, 2009, p.13) "es un proceso de transformación en la cual las escuelas regulares desarrollen respuestas a la diversidad del alumnado que tienen escolarizado, identificando y eliminando las barreras que este entorno pone a su aprendizaje, socialización y participación". Además, no solo es el deber de las escuelas de educación especiales velar por los niños (as) con dificultad físicas e intelectual, sino que también es la responsabilidad y obligación de las escuelas regulares aceptar y dar la oportunidad a cualquier niño o niña en dichas condiciones para convivir con sus iguales y desenvolverse en cualquier actividad escolar, porque la escuela también es un medio para favorecer en su desarrollo cognitivo, intelectual, motriz etc.

El estudio cualitativo realizado en las escuelas o aulas nos puede proporcionar una doble ventaja, aprender sobre ellas de manera que sea útil para comprender otras escuelas y aulas respecto de las situaciones que viven actualmente los profesores/as acerca de la inclusión de educandos indígenas y con discapacidad. 
Se enmarcó el estudio desde el paradigma cualitativo con enfoque comprensivo interpretativo, pues se trató de indagar una problemática cargada de significaciones personales, sociales, culturales, ideológicas, valorativas desde las perspectivas que poseen los profesores indígenas sobre su propia praxis, desde un estudio de caso, tipo intrínseco. Su propósito básico es alcanzar la mayor comprensión del caso en sí mismo. Queremos aprender de él en sí mismo sin generar ninguna teoría ni generalizar los datos (Stake, 1999).

Para la selección de las dos escuelas primarias indígenas, se realizó una exploración previa para conocer que escuelas habían tenido mayor matrícula de alumnos (as) con dificultades motrices y cognitivas para describir y comprender mejor el fenómeno educativo que se daba desde la experiencia y visión del docente el contexto áulico. La muestra de carácter estructural, se seleccionaron 8 profesores de dos escuelas primarias indígenas. Entre ellos, dos directivos y seis docentes de aulas. Todos ellos hablantes de lengua indígena, originarios de la región de mixteca, experiencia laboral de 5 a 15 años, entre 28 y 45 años de edad, de ambos sexos. La técnica utilizada para esta recabar los discursos fue la entrevista en profundidad, para asegurar la credibilidad se optó por la realización de un proceso de triangulación de carácter teórico.

A continuación se presentan las categorías construidas a partir de los discursos de los docentes indígenas.

\section{La representación de los docentes sobre su formación para atender a niños y niñas en situación de discapacidad}

Los profesores indígenas enfrentan un conjunto de desafíos; se les pide establecer interrelación entre su cultura de origen y la cultura nacional; son docentes bilingües con diversos niveles de competencia lingüística tanto en español como en alguna de las lenguas indígenas; tienen distintos niveles de conocimiento, tienen necesidades y expectativas de superación profesional diversas, la formación que han recibido para ejercer la docencia es diferente, pues no siempre se ha vinculado con los problemas que enfrenta en su práctica profesional.

Por lo tanto para atender las necesidades pedagógicas de los alumnos con discapacidad en sus diferentes modalidades, físicas cognitivas, sensoriales, es necesario de una formación inicial docente y continua. Sin embargo, hay obstáculos que impiden el acceso a los diferentes cursos, talleres, diplomados que las diferentes instituciones públicas y privadas ofertan como; la lejanía del espacio laboral de los docentes, la carga laboral, lo económico, el factor tiempo entre otros.

"No he recibido cursos para trabajar con los chiquitos, (...) lo que me ha obligado averiguar en internet a leerle, a investigar, entonces lo poquito o mucho que sé, es por experiencia laboral, pero de que me hayan dado algún cursito o taller sobre el tema no, algunas cosas lo he tenido que aprender por otros medios" (D1). 
Los profesores sostienen que no tienen una formación ni están capacitados para abordar la discapacidad en el aula, eso genera en ellos una fuerte tensión que incluso los lleva a sentir incapacidad e irresponsabilidad profesional frente a la discapacidad del alumno. Dicha incapacidad está dada y se manifiesta en: una falta de manejo de metodologías y estrategias pedagógicas.

Por otra parte, hay docentes quienes señalan que tomar cursos sobre atención a niños con discapacidad solo les compete a los docentes que trabajan en centros de educación especial, si bien, en diferentes leyes y programas se menciona que todos los profesores que atienden escuelas regulares <normal> deben estar preparados para atender la diversidad que haya en su espacio educativo.

La formación de un docente indígena, es relevante dado que les permitirá adquirir habilidades para afrontar los problemas y situaciones que se generan en su espacio educativo. Así como todo docente debe tener compromiso por el aprendizaje de los niños (as), dominio de las didácticas temáticas y de diferentes modelos de enseñanza, capacidad de colaborar con otros docentes y la capacidad de reflexión para poder construir alumnos críticos y responsables para la sociedad del conocimiento y en constante trasformación.

\section{La representación de los docentes sobre su práctica pedagógica para trabajar con niños (as) con dificultades motrices y cognitivas}

El docente desde su desempeño, como mediador y formador, señala que reflexiona sobre su práctica pedagógica, para mejorarla, fortalecerla y elaborar nuevos conocimientos, por ello consideran que parte de su ejercicio profesional consiste en seguir enseñando y construir saberes pese a las situaciones particulares de cada aula, si bien, cada docente significa de forma particular las diferentes situaciones que existe en su espacio escolar.

Por ello, desde Blumer: "Entiende que el ser humano orienta sus actos hacia las cosas en función de los que estas significan para él, el significado de las cosas se deriva a través de la interacción social que mantienen con el otro. De tal manera que estos significados se manipulan y modifican mediante un proceso de interpretativo desarrollado por la persona al enfrentarse con las cosas que va hallando a su paso. (Blumer, 1982, p. 2).

Con lo antes expuesto, los profesores indígenas de las escuelas regulares construyen e interpretan la realidad del aula desde sus propias cosmovisiones, historias y vivencias, de esta manera orientan su trabajo pedagógico con los alumnos con dificultades físicas y cognitivas con lo que éstos significan para ellos y la construcción intersubjetiva que realizan producto de los diversos modos de comprender, relacionarse y de la interacción cotidiana dentro del aula con dichos estudiantes. Los significados que emergen del encuentro diario, se mantendrían a través de las rutinas que se podrían ir modificando de acuerdo a la interpretación y reflexión que el propio docente realiza de su práctica. 
"He trabajo con varios niños con capacidades diferentes a lo largo de estos años, pero no he hecho ninguna adecuación, trato de hacer lo que puedo para trabajar con ellos y también lo que ellos logren aprender, tampoco puede exigirle al niño enfermo, lo que un niño sano puede hacer, seria inadecuado". (D1).

Desde esta postura el docente significa en su discurso no tener una formación para atender al alumno con dificultades, sin embargo, él revela que realiza todo lo que esté a su alcance para lograr comprender las situaciones, como un compromiso moral que tienen con el estudiante, porque considera elementos intuitivos y de creatividad para generar espacios de socialización y el desarrollo de actividades pedagógicas para lograr el aprendizaje de cada uno de los estudiantes con discapacidad.

"Cuando veo que el alumno se atrasa mucho con las actividades me quedo con él unos 10 minutos después de clases, para explicar las diferentes actividades desarrolladas y decirle de que manera puede seguir trabajando en casa" (D2).

La situación de los docentes indígenas frente a esta situación resulta complejo, desde esta argumentación se puede interpretar que a través de los años algunos profesores han significado de diferente modo y la forma a través de sus experiencias, acerca del tipo de estrategias a implementar para trabajar con cada estudiante con diferentes dificultades, considerando el ritmo de aprendizaje y capacidad, además algunos profesores se ven comprometidos más que otros por lo que le suponen tiempo extra para ayudarlos con las materias vistas y retroalimentar lo aprendido en clases.

"Lo pongo a trabajar con un niño más inteligente que él y a ese niño le pido que por favor lo apoye es como decimos nosotros en teoría, un experto con un novato para que ahí se vaya compensando el aprendizaje" (D2).

Una docente de la muestra señala que es complicado darle atención especial a cada niño o niña con discapacidad más cuando se encuentra inmerso con un grupo grande, y la estrategia que ella implementa para ayudarse en la atención con estos niños, es pedir apoyo a un estudiante aventajado sin discapacidad para apoyar al alumno con discapacidad, para que estos niños avancen con las diferentes actividades que se desarrolla en las clases.

Desde estas posturas los docentes significan su labor pedagógico como algo difícil porque implica más demanda de tiempo para atender las necesidades de cada alumno, y esto le genera conflictos pese a que el docente tienen que cumplir su planeación y responder a los lineamientos que señala el currículo nacional.

\section{La representación que tienen los docentes sobre la causa de la discapacidad}

El docente de educación indígena tiene una forma peculiar ver y entender las diferentes situaciones que aquejan su espacio educativo, tal como las causas de la 
discapacidad, pues si bien son temas que la mayoría de los docentes ha enfrentado en el trayecto de su experiencia laboral y que han tenido que apoyar al alumno y a la familia.

Una vez que el docente indígena parte de la realidad en la que se encuentra sumergido su espacio áulico, identifica el tipo y la causa de la discapacidad que puedan tener algunos alumnos presentes dentro del salón de clase. Desde la perspectiva de lo concebido desde la cultura a la cual pertenecen, o lo que han aprendido, diagnosticado, o averiguado con las familias de los niños con dificultades.

Así, la causa de la discapacidad es atribuida a diferentes factores: social, cultural, económica, desde lo sobrenatural hasta congénita, hay quienes presentan una discapacidad al nacer por diferentes enfermedades, una mala alimentación de la madre durante el embarazo, el consumo de alcohol, la violencia doméstica que sufre la mujer durante el embarazo, asimismo la discapacidad también es generada por accidentes en el trayecto del crecimiento de un niño o niña; la mayoría de los accidentes en las comunidades indígenas son por el descuido de la crianza.

Ante esto, un profesor expresa en su discurso conocer la causa del padecimiento que tienen sus alumnos.

“Este niño tenía una hidrocefalia, una parálisis de lado derecho de su cuerpo, era lo que sabíamos era lo que los familiares comentaban, pues, había limitante en el habla y también motriz, tenía el brazo derecho y la pierna derecha una rigidez completa, era muy difícil que él tuviera movimientos en esas parte del cuerpo prácticamente era de su lado de derecho; el otro niño fue un problema al nacer, al parecer fue el cordón umbilical el que le hizo presión y pues lo lastimó neurológicamente" (D1).

Asimismo, dentro de la cultura ñuu savi hay cosmovisiones que giran en torna a estos padecimientos especialmente asociada a la discapacidades intelectuales y cognitivas. Se considera que es un problema surgido de la brujería que podría deberse a la envidia el mal del prójimo, castigo de Dios, la enfermedad del espanto, básicamente es la relación del hombre con todo su entorno y ante el incumplimiento de alguna norma a la naturaleza o algún ente natural o sobrenatural es enviado una señal a través de enfermedades que limitan parte del cuerpo y la cabeza del ser humano.

“Ahora si sabrá Dios son cuestiones congénitas o algún mal que haya recibió él o su mamá y que lo heredado el niño, solo el destino sabe, porque vienen con esa discapacidad, pobrecitos veo porque aquí tuvimos un muchacho en el periodo antepasado que tenía una discapacidad le costaba mucho hacer cosas en la escuela" (D5).

Desde esta posición los docentes han tenido que hacer de médico, a través de su experiencia; han tenido que diagnosticar intuitivamente el padecimientos de sus alumnos 
con dificultades motrices y cognitivas para que los padres logren también comprender la naturaleza de la enfermedad y de esta manera ayudar en su proceso de aprendizaje.

El rol del docente no solo es cumplir los planes y programas que se les pide, sino también que tienen que hacer otras actividades para poder apoyar a los alumnos con discapacidad, están al pendiente de sus alumnos, procurando saber sobre sus padecimientos o enfermedades para poder apoyar o guiar a los padres para buscar apoyos en otras instituciones.

\title{
4. La representación que tienen los docentes sobre las características de los alumnos que padecen dificultad motriz e intelectual
}

Para los docentes de la comunidad de región ñuu savi las dificultades físicas y cognitivas son los más visibles en tanto que repercute en sus diferentes formas de discriminación para poder acceder a los diferentes espacios de socialización y educativo tal como podemos ver en el siguiente discurso del docente.

\begin{abstract}
"Al principio en su ingreso en la escuela de los dos niños uno que estaba enfermo de la cabeza y el otro que estaba mal del cuerpo fue como oyente, porque [vino] la mamá a pedir que se aceptara su hijo y el otro lo trajo sus abuelitos, pero la verdad la escuela no quería aceptar a los niños porque pensaban que tenían algo contagioso o algún mal, porque pues uno de los niños babeaba, y caminaba mal, pero después de tanta insistencia lo aceptaron y les dieron la oportunidad de estar en la escuela como oyente a los dos, y así fue como poco a poco lo fueron aceptando fueron integrándose (...) poco a poco se fue viendo que nada era contagioso y se aceptó a esos niños pero pues es mucho trabajo porque muchos de los profesores no saben qué hacer y a veces van pasando de grado sin que aprendan". (D7).
\end{abstract}

Debido a las características que presentan los niños y niñas con discapacidades intelectuales y cognitivas, muchas veces la comunidad educativa genera rechazo para estos alumnos porque consideran que no tienen ningún caso que se les dé la oportunidad de estar en la escuela que ya probablemente no podrán aprender, por las diversas razones que giran en torno a las dificultades.

Desde el discurso los docentes señalan que si los alumnos que se ven físicamente graves puede que no se otorgue un espacio dentro de la escuela, si se llega a lograr que el alumno con discapacidad se incorpore es porque los padres quieren que sus hijas aprendan y son insistentes ante esta apertura y para no tener problemas mayores deciden aceptar al alumno, pero solo como oyente sin el compromiso sobre su aprendizaje.

A su vez el docente caracteriza a los alumnos con dificultad en base a sus capacidades, comportamiento, nivel de aprendizaje, participación y el desarrollo de las 
diferentes actividades educativas que considera que puede lograr a ejecutar y la socialización con el resto del grupo.

Por su parte, los profesores consideran que depende de una gran variedad de factores, para lograr que estos alumnos con dificultad motriz y cognitiva puedan tener una mejor participación en el espacio escolar, muchos de los cuales son externos e imposible de cambiar por el contexto en el que se encuentran.

\section{La representación de los docentes sobre la situación familiar de los de niños (as) que padecen dificultades físicas y cognitivas}

En la comunidad Nuu Savi existen diferentes actitudes hacia los niños con alguna discapacidad, estas diferencias se observan en actitudes culturales y comunitarias que tienen las familias frente a los niños (as) con dificultades cognitivas y motrices, las construcciones que tienen a cerca del fenómeno pueden variar de una familia a otra, pues hay familias más abiertas, solidarias y respetuosas, ello incide en las relaciones familiares, en cómo perciben y resuelven los problemas.

Para los pueblos originarios tener algún integrante en la familia con algún tipo de discapacidad, resulta una situación compleja por las condiciones de marginación en las que se encuentran la mayoría de las familias, dada la inadecuada atención que ofrecen los centros de salud y la educación que el Estado proporciona y aunado a la falta de empleo, muchas veces las personas tienen que salir a buscar mejores oportunidades a otras países o ciudades para poder cubrir las necesidades básicas.

Para lograr cubrir los gastos que implican la atención y el cuidado de un hijo con discapacidad, muchas veces algún familiar tiene que salir a buscar empleo en otros espacios fuera de la comunidad. Ante esto es común ver a niños con discapacidad que viven con los tíos, abuelos o los hermanos mayores, ello implica que el proceso de cuidado y la atención en su desarrollo de aprendizaje sean descuidados.

"Estamos en una comunidad rural, donde se viven muchas problemáticas, y uno de ellos es lo económico porque los papás que se van en busca de una mejor vida y dejan a sus hijos, y los perjudicados al final son los niños porque no reciben mucha atención, es difícil muy difícil, que quisiéramos que los niños tuvieran de todo pero es difícil por el contexto en el que viven" (D2).

Hay que mencionar además, que en estas comunidades indígenas aún hay otros factores que afectan a los niños con dificultad para su protección y su paso por la escuela tal como se señala la profesora en su discurso;

"El factor familiar es otro problema, parece ser que el papá es un poco agresivo con la mamá, y esto influye también causa consecuencias en los alumnos 
enfermos, entonces le digo hay variedad de factores que influyen en el aprendizaje de los niños" (D3).

Desde lo que se infiere en los discursos de los docentes la labor familiar en la inclusión y el aprendizaje del niño con dificultad es de suma importancia para su progreso. Se considera la familia uno de los principales recursos de apoyo para lograr progresos en los aprendizajes de los estudiantes, por tanto elle debe participar e involucrarse en la toma de decisiones educativas respecto de sus hijos con dificultad, construyendo con ella una relación positiva y de colaboración con los docentes para observar los aprendizajes y progresos del estudiante, mientras más involucrados están los padres en el aprendizaje y en los procesos educativos que viven sus hijos(as), mayores logros pueden alcanzar los estudiantes.

Los padres de los niños con discapacidad pueden jugar un papel principal en su educación, si se les facilita y permite. Este papel puede incluir la colaboración en las actividades de la escuela, apoyar programas en el hogar y controlar el progreso de los estudiantes (Hegarty, 1994). Asimismo, constituyen los primeros y naturales profesores del niño, por lo que tiene sentido ayudarles a desempeñar este papel con lo mejor de sus potencialidades. Aun representa una limitante para las familias poder apoyar a los hijos con alguna discapacidad porque hay muchos factores que impiden este proceso.

\section{La representación de los docentes sobre los niños (as) que padecen algún tipo de dificultad que no están integrados en la escuela}

Desde lo que plantea el modelo social de la discapacidad, que las niñas y niños con discapacidad son sujetos de derecho, por lo tanto tienen las mismas oportunidades de desarrollo que los niños (as) sin discapacidad. Esto incluye el acceso a muchas áreas, como la educación, y una plena inclusión en las diferentes actividades pedagógicas, juegos, deportes, bailes culturales y demás, estos espacios deben encontrarse en condiciones de poder ser aprovechadas por niñas (os) con discapacidad, en igualdad de condiciones que el resto. Es decir, todas aquellas actividades que resultan ser imprescindibles para el desarrollo tanto físico, como psicológico y social de las niñas (os) con y sin discapacidad.

"Aquí entra el papel de nuestra autoridad a lo mejor ya no de la escuela, ellos son responsable para eso está nuestro regidor de educación buscar a todos los niños para que se incorporen a la escuela es un derecho de los niños recibir educación, y no dejarlos a un lado, porque si no es una discriminación por sus características y no debe de ser así, porque ellos deben buscar a todos, deberían tener un censo de esas niñas y niños que están con esas características para que se integren a la sociedad, (...) porque la escuela enseña mucho cantar, bailar, reír, corre, y muchas cosas y esto recrea la mente de los niños" (D 8).

Desde esta postura el docente señala que: el Estado es la primera instancia quien debe velar por todos los alumnos con discapacidad, al mismo tiempo tiene claro que estos niños 
en estas condiciones tienen la misma oportunidad de acceder a cualquier sistema educativo, reconocen como un derecho fundamental que tienen las niñas y niños. Que es la responsabilidad de todos los agentes que viven en la comunidad y de los actores educativos llevar a cabo acciones que generen la identificación temprana de los niños con discapacidad para abrir espacios de socialización en los diferentes contextos.

\section{CONCLUSIONES}

Para comprender las representaciones de los docentes a su práctica pedagógica con niños (as) con dificultad física y cognitiva en dos escuelas de educación primaria indígena, se indagaron en las experiencias de los docentes: su formación y desempeño profesional frente a la discapacidad; las dificultades que tienen ante el desafío de tener alumnos en dichas condiciones en el espacio áulico regular.

El análisis de los datos y los diferentes discursos que emergieron develaron que: cada uno de los profesores indígenas de las escuelas regulares, está cargado de experiencias personales que dan significado a su labor pedagógica, esto confluye en su forma de socializar, entender y tratar a los niños con discapacidad, así como la manera de ayudar tanto al niño como a su familia para poder sobrellevar la situación dentro y fuera de la escuela.

En relación con la identificación de cómo los profesores nombran la causa de la discapacidad desde la cultura, los significados que han construido sobre este fenómeno se encuentra enraizado con su cosmovisión y la experiencia que ha tenido a través de los años para detectar y diagnosticar las causas de las diferentes discapacidades. Asimismo el origen de algunas dificultades que les diagnosticaban a los alumnos señalaban que eran por factores congénitas, accidentes y por razones de orden natural o sobrenatural (divinos), según su cultura ñuu savi.

En cuanto a la característica de la práctica, la mayoría de los profesores significan que aprendieron a trabajar con estos niños y niñas desde lo que para ellos significaba la condición de cada alumno y de sus perspectivas capacidades para desarrollar las actividades, señalando que a lo largo de sus experiencia han tenido que trabajar a través de prácticas intuitivas y tradicionalistas, pues consideraban que era la única forma que conocían para apoyar a los alumnos, si bien algunas prácticas que utilizaban violentaba al alumno con dificultad para su proceso de aprendizaje, por imponer una planeación sin una previa adecuación y sin considerar las actitudes y aptitudes de los alumno para desarrollar ciertas actividades.

Asimismo, los profesores significan desde sus experiencias que prefieren que estos alumnos asistan a las escuelas especiales, considerando que están dotados de todos los materiales y que son especialmente para atender a los alumnos con dificultad y que probablemente por tener personal especializado darán una mejor atención, versus a la atención que ellos le proporcionan, dado que la escuela primaria indígena regular los 
grupos son numerosos por lo que la atención de los alumnos resulta más demandante y esto genera conflictos para ellos darles atención especial o preparar material con adecuaciones para el niño con discapacidad. Por lo que consideraban una labor difícil de llevar a cabo en todos los sentidos.

En referencia a las competencias de los docentes en el trabajo con los alumnos con dificultad física y cognitiva, significan que es un reto día con día, cuando al inicio de cada ciclo escolar tienen un nuevo alumno con dichas condiciones porque las demandas del sistema educativo va cambiando cada año y ello también su rol como profesor por los nuevas problemáticas que pueda presentar su grupo escolar. Aunque señalan que siempre deben estar en la mejor disposición para cualquier nuevo reto que pueda presentarse en la escuela. Al mismo tiempo, manifiestan sobre la apertura de espacios de profesionalización en diferentes temáticas pero con énfasis especial en métodos o estrategia para trabajar con niños con discapacidad, que les permita conocer y poder desarrollar una planeación adecuada para la atención de cada uno de los alumnos con dificultad, de esta forma puedan ayudar y guiar al alumno en su aprendizaje.

Del mismo modo, señalaron que hay otros factores que impiden una plena inclusión y el avance del trabajo con los alumnos con discapacidad, expresando que, los padres son los principales responsables de la educación de sus hijos así como parte importante de este proceso de aprendizaje, sin embargo encuentran que por las condiciones sociales y económicas en las que se encuentran, muchos de ellos descuidan el proceso de aprendizaje-enseñanza, lo que conlleva que no se pueda llevar un trabajo colaborativo escuela-familia para el progreso de cada uno de los alumnos.

El panorama general de las condiciones en las que se encuentran las dos escuelas primarias indígenas regulares y de la formación de los docentes indígena en la atención a los niños con dificultad física y cognitiva como una primera barrera para la labor docente. Por cuanto, ninguna de las escuelas cuenta con infraestructura adecuada para recibir y atender a los niños con discapacidad como, sillas, mesas, el acceso de sillas de rueda, ni materiales pedagógicos. Considerando que la institución educativa es la que debe adaptarse para recibir a todo tipo de estudiantes, y no que los alumnos se adecuen a las escuelas.

Hay carencia de la formación de los profesores indígenas para atender a la diversidad de alumnos con dificultad, esto significa que repercute en la preparación de una planeación y adecuación de estrategias pedagógicas para trabajar con los alumnos, la mayoría de los profesores consideraba que, los alumnos con discapacidad tenían que adecuarse a las actividades que trabajaban con el resto del grupo.

Ello exige de un docente indígena una actitud proactiva, con una práctica pedagógica reflexiva y flexible que respete las diferencias y las aproveche para propiciar el enriquecimiento colectivo de la diversidad en su espacio escolar, que genera expectativas 
positivas y flexibles hacia niñas y niños con discapacidad intelectual y cognitiva lo cual permita favorecer su formación integral.

Finalmente, los docentes reflexionan en torno a las acciones que el gobierno federal, estatal y local de tomar para la inclusión plena de los niños con discapacidad, si bien como ellos planteaban que es el derecho de todo ser humano lograr el acceso a la educación, bajo estas premisas, las escuelas de educación indígena han de acoger, desde esta concepción, a los alumnos/alumnas con discapacidad así como incluir al trabajo del aula los conocimientos de los pueblos originarios. La diversidad en el salón de clases y en el marco de la educación inclusiva se debe abrir a todas las posibilidades para que el profesor crezca profesionalmente, lo motive a buscar soluciones a las necesidades del alumnado, a modificar su práctica en el aula, a crear estrategias para afianzar valores, a encontrar la manera de involucrar a madres, padres y a otros agentes educativos comunitarios en los logros educativos de todos los alumnos/alumnas con y $\sin$ discapacidad, es decir, valorando al alumnado por sus capacidades y sus posibilidades, es concebir la inclusión como una oportunidad de crecimiento para todos.

\section{REFERENCIAS BIBLIOGRÁFICAS}

Blumer, H. (1982). El interaccionismo simbólico. Barcelona: Hora. S.A.

Delgado, J. M. y Gutiérrez, J. (1995). Métodos y técnicas de investigación cualitativa en ciencias sociales. Madrid: Síntesis.

Delors, J. (1996). La educación encierra un tesoro. España: Santillana.

DOF (2015). Ley general para la inclusión de las personas con discapacidad. México.

DOF (2016). Constitución Política de los Estados Unidos Mexicanos. México.

Guerra, M. (2006). La escuela que aprende. Madrid: Morata.

Hegarty, S. (1994). Educación de niños y jóvenes con discapacidades principios y práctica. UNESCO.

INEGI (2013). Las personas con discapacidad en México, una visión al 2010. Blog post http://internet.contenidos.inegi.org.mx/contenidos/productos//prod_serv/contenidos/es panol/bvinegi/productos/censos/poblacion/2010/discapacidad/702825051785.pdf

Macarulla, I. y Saiz M. (2008). Buenas prácticas de escuela inclusiva. La inclusión de alumno con discapacidad un reto, una necesidad. Barcelona: GRAÓ.

Mucchielli, A. (1996). Diccionario de métodos cualitativos en ciencias humanas y sociales. Venezuela. 
Palacios, A. (2008). El modelo social de discapacidad: orígenes, caracterización y plasmación en la convención internacional sobre los derechos de las personas con discapacidad. Madrid: CINCA.

Pérez, A. (2000). La cultura escolar en la sociedad neoliberal. Madrid: Morata.

Soto, A. (2011). La discapacidad y sus significados: notas sobre la (in)justicia. Política y Cultura 35: 209-239. http://www.redalyc.org/articulo.oa?id=26718442011 